\section{Validade do auto-relato de diabete mellitus gestacional no pós-parto imediato}

\author{
Validity of self-reported gestational diabetes \\ mellitus in the immediate postpartum
}

\author{
1 Faculdade de Medicina, \\ Universidade Federal de \\ Pelotas, Pelotas, Brasil. \\ Correspondência \\ M. A. S. O. Dode \\ Centro de Pesquisas \\ Epidemiológicas, Faculdade \\ de Medicina, Universidade \\ Federal de Pelotas. \\ Rua Padre Anchieta 2023 \\ apto. 801, Pelotas, RS \\ 96015420, Brasil. \\ malicedode@terra.com.br
}

\begin{abstract}
Studies based on self-reporting of risk factors or morbidity are useful in public health, due mainly to the low cost and ease of data collection, but the accuracy of the resulting data is important for the internal validity of the research. The aim of this study was to evaluate the agreement between self-reported gestational diabetes mellitus and prenatal care medical records (gold standard). Agreement was measured by sensitivity, specificity, accuracy, kappa statistic, and confidence intervals (95\%CI). From July to December 2007, all the women who gave birth in three maternity hospitals in Pelotas, Rio Grande do Sul, Brazil, were visited in the immediate postpartum. 1,047 women were interviewed, of whom 872 were in possession of their prenatal care card; of these, 869 had their blood glucose recorded on the card. Mean age was 26.7 years. Prevalence of gestational diabetes mellitus based on the gold standard was 4.3\% (95\% CI: 3.0-5.8), while the self-reported rate was 4\% (95\%CI: 2.8-5.5). Sensitivity of selfreported gestational diabetes mellitus was $72.9 \%$ (95\% CI: 55.9-86.2); specificity was 99\% (95\%CI: 98.1-99.6); accuracy 97.9\% (95\%CI: 96.7-98.7); kappa $74 \%$. For this population, self-reported gestational diabetes mellitus is valid information and can be used in population-based studies.
\end{abstract}

Gestational Diabetes; Maternal Welfare; Prevalence
Maria Alice Souza de Oliveira Dode 1

Iná S. Santos 1

\section{Introdução}

A prevalência e a distribuição de determinados agravos à saúde são frequentemente obtidas por meio de inquéritos populacionais $1,2,3,4,5$. Os inquéritos de saúde utilizam quase que exclusivamente questionários como instrumento de coleta de dados. Mas a obtenção de determinadas mensurações pode necessitar, por vezes, de equipamentos específicos, como aparelhos de medida de pressão arterial, estadiômetros, balanças, glicosímetros, e outros mais sofisticados. A coleta dessas medidas de maneira direta, seguindo as padronizações específicas, melhora a qualidade da informação e a validade interna do estudo. Contudo, as limitações de custo para aquisição de aparelhos, a dificuldade de transporte e a complexidade de realização de testes e medidas podem tornar difícil a operacionalização do campo. Como conseqüência, muitos estudos utilizam informações auto-referidas.

Na literatura, diversos estudos conduzidos em diferentes grupos populacionais utilizaram o diagnóstico auto-referido de diabetes mellitus $4,5,6,7,8,9,10,11,12$. As perguntas frequentemente empregadas para identificar diabetes mellitus autoreferido são: "Algum médico lhe disse que o $\operatorname{Sr}(a)$ tem diabetes?", "Alguma vez o Sr(a) teve diabetes?" ou simplesmente "O(A) Sr(a) tem diabetes?". $\mathrm{O}$ uso desse tipo de medida permite que sejam estudadas grandes amostras populacionais com maior rapidez e menor custo 7,12,13. 
O diabetes mellitus gestacional é uma condição caracterizada por intolerância a glicose, em variados graus de intensidade, com início ou diagnosticada pela primeira vez na gravidez 14 . No Brasil, o Ministério da Saúde recomenda que todas as gestantes sejam rastreadas para diabetes mellitus gestacional na primeira consulta prénatal, através de glicemia de jejum, sendo este repetido na vigésima semana. Se a glicemia for superior a $90 \mathrm{mg} / \mathrm{dL}$ e encontrar-se na faixa entre $\geq 90 \mathrm{mg} / \mathrm{dL}$ e $\leq 109 \mathrm{mg} / \mathrm{dL}$, a gestante deverá ser encaminhada para um teste de sobrecarga de duas horas, com $75 \mathrm{~g}$ de glicose. O teste de sobrecarga deve ser realizado entre as 24a e 28a semanas de gestação. As glicemias diagnósticas de diabetes mellitus gestacional são $\geq 110 \mathrm{mg} / \mathrm{dL}$ no jejum, $\geq 140 \mathrm{mg} / \mathrm{dL}$ duas horas pós sobrecarga ou, em qualquer momento, acima de $200 \mathrm{mg} / \mathrm{dL}$. A glicemia de jejum $\geq 110 \mathrm{mg} / \mathrm{dL}$, na vigésima semana, após repetição, também confirma o diagnóstico 15

A revisão da literatura, conduzida nas bases de dados PubMed, LILACS, SciELO, assim como no banco de teses da Coordenação de Aperfeiçoamento de Pessoal de Nível Superior (CAPES), não identificou a existência de estudos que tenham avaliado a acurácia do auto-relato de diabetes mellitus gestacional em população brasileira. O objetivo deste estudo foi investigar a validade do auto-relato da presença de diabetes mellitus gestacional no pós-parto imediato.

\section{Métodos}

O presente trabalho é resultado de um estudo transversal realizado durante os meses de julho a dezembro de 2007, nas maternidades dos hospitais Santa Casa de Misericórdia de Pelotas, Hospital Universitário São Francisco de Paula e Hospital Miguel Piltcher, onde ocorrem aproximadamente $70 \%$ dos nascimentos da cidade de Pelotas, Estado do Rio Grande do Sul, Brasil.

Logística, instrumento, definição operacional do desfecho e padrão-ouro

Diariamente, nas primeiras 24 horas do pós-parto, seis estudantes de Medicina realizaram as entrevistas nos três hospitais, utilizando questionários padronizados e pré-codificados. O diagnóstico de diabetes mellitus gestacional auto-referido foi obtido mediante duas perguntas: “Durante essa gravidez a Sra teve diabetes?”. Em caso de resposta afirmativa: "Já tinha diabetes antes da gravidez?".

Foram consideradas portadoras de diabetes mellitus gestacional auto-referido as mães que responderam afirmativamente à primeira pergunta e negativamente à segunda. O padrão-ouro utilizado foi o registro na carteira da gestante do resultado de glicemia ou teste de tolerância a glicose, feito pelo médico, durante o pré-natal. Se, em vez do registro na carteira da gestante, as mães portassem resultados de exames de glicemia feitos durante a gravidez, estes eram também anotados como padrão-ouro. Foi considerada com diabetes mellitus gestacional a gestante cujo registro na carteira mostrou glice$\mathrm{mia} \geq 110 \mathrm{mg} / \mathrm{dL}$, confirmado por repetição, e/ou glicemia duas horas pós-carga de 75 g glicose $\geq$ $140 \mathrm{mg} / \mathrm{dL}$.

Foram ainda coletadas informações sobre a idade, cor da pele, peso pré-gestacional e altura auto-referidos, assim como peso pré-gestacional e altura anotados no cartão da gestante. Foi utilizada a informação de peso pré-gestacional registrado na carteira de gestante somente quando esse fora obtido nos três primeiros meses de gestação. A idade foi coletada em anos completos por ocasião do parto e posteriormente categorizada em $\leq 19$, 20-29 e 30 anos ou mais. A cor da pele foi obtida por observação dos entrevistadores e classificada como branca, negra e parda. $\mathrm{O}$ índice de massa corporal (IMC) pré-gestacional foi calculado através da divisão do peso/quadrado da altura e, após, categorizado em baixo peso $(<20)$, normal $(20<25)$, sobrepeso $(25<30)$ e obesidade $(\geq 30)$.

\section{Cálculo de tamanho de amostra}

Através dos dados da coorte de nascimentos de Pelotas de 2004 16, obteve-se a informação de que nascem na cidade cerca de 300 crianças ao mês. O tamanho da amostra foi calculado estimandose que $80 \%$ dos médicos solicitassem glicemia de jejum ou teste de sobrecarga com glicose, e que a prevalência de diabetes mellitus gestacional fosse de cerca de $4 \%$ a $8 \% 17$. Em seis meses de trabalho de campo, seriam entrevistadas aproximadamente 1.260 puérperas, 50 a 100 das quais teriam diabetes mellitus gestacional. Com esse número de mães, seria possível detectar uma sensibilidade de $80 \%$ a $85 \%$, com erro de $\pm 0,15$, com $95 \%$ de confiança.

\section{Análises estatísticas}

Foi realizada dupla digitação dos dados. A validação da digitação foi feita por intermédio da função validate do programa Stata (Stata Corp., College Station, Estados Unidos). Para as análises, utilizou-se tabela $2 \times 2$, em que a resposta da mãe foi comparada ao padrão-ouro. Sensibilidade, especificidade, valores preditivos positivo e 
negativo (VPP e VPN) e a acurácia da informação da mãe foram calculados utilizando-se fórmulas específicas.

A sensibilidade foi o quociente da divisão do número de puérperas que relataram acertadamente ter diabetes mellitus gestacional pelo total de puérperas da amostra que tinham efetivamente a doença de acordo com o padrão-ouro. A especificidade foi obtida pela divisão do número de puérperas que relataram acertadamente não ter diabetes mellitus gestacional pelo total de puérperas na amostra que efetivamente não tinham a doença. O VPP foi o quociente das que declararam ter diabetes mellitus gestacional e que de fato a tinham dividido por todas as que declararam ter; e o VPN, o quociente das que declararam não ter diabetes mellitus gestacional e que efetivamente não tinham, dividido pelo total das que declararam não ter a doença. A acurácia foi o resultado da soma das que declaram acertadamente ter diabetes mellitus gestacional e não ter diabetes mellitus gestacional dividido pelo total de mulheres na amostra.

Utilizou-se a estatística kappa para avaliar a concordância entre o auto-relato de diabetes mellitus gestacional e o padrão-ouro, descontando o acaso. Para este cálculo, empregou-se o programa Stata para os dados registrados; para os dados expandidos, o cálculo foi realizado a mão. VPP e VPN para diferentes prevalências de diabetes mellitus gestacional foram estimados através do teorema de Bayes segundo as fórmulas:

$$
\begin{aligned}
& \text { VPP }=\text { prevalência } \mathrm{x} \text { sensibilidade } \\
& \text { (prevalência x sensibilidade) + } \\
& \text { ( } 1 \text { - especificidade) ( } 1 \text { - prevalência) }
\end{aligned}
$$

Para investigar se fatores maternos poderiam afetar os resultados, realizou-se análise de regressão logística, em que a variável dependente foi definida como "concordância" (registro e auto-relato concordantes).

Foram calculados intervalos de 95\% de confiança (IC95\%) para todos os parâmetros avaliados. O projeto foi aprovado pelos Comitês de Ética e Pesquisa dos respectivos hospitais. Todas as entrevistas foram realizadas após consentimento verbal informado.

\section{Resultados}

Um total de 1.052 mulheres era elegível para o estudo. Cinco recusaram-se a responder a en- trevista, restando 1.047. Entre as entrevistadas, 872 estavam com a carteira pré-natal, das quais 869 continham registro de glicemia, sendo estas incluídas na investigação. A prevalência de diabetes mellitus gestacional conforme registro na carteira pré-natal (padrão-ouro) foi de 4,3\% (IC95\%: 3,0-5,8) e conforme o auto-relato, de $4 \%$ (IC95\%: 2,8-5,5).

A média de idade encontrada foi 26,7 anos, variando de 13 a 49 anos. A média de peso prégestacional auto-referido foi de 61,6kg (desviopadrão $-\mathrm{DP}=12,7 \mathrm{~kg}$ ) para um n de 963 puérperas. A média de peso medido nos primeiros três meses foi de $64,9 \mathrm{~kg}$ ( $\mathrm{DP}=14,2 \mathrm{~kg}$ ); ressalte-se que, para esta medida, contava-se com a informação no cartão de gestante de somente 494 puérperas. As médias de peso pré-gestacional registrado e auto-referido foram estatisticamente diferentes $(\mathrm{p}<0,001)$. Por outro lado, com relação aos dados de altura, praticamente não houve diferença entre os valores registrados e os auto-referidos (respectivamente, $1,60 \pm 6,6 \mathrm{~cm}$ e $1,61 \pm 6,9 \mathrm{~cm}$; $\mathrm{p}=0,18)$.

Não houve diferença significativa na distribuição de idade, cor da pele e IMC pré-gestacional (calculado com base no peso auto-referido), além de hospital de origem, entre as mulheres que levaram ou não a carteira pré-natal para o hospital (Tabela 1).

A Tabela 2 mostra os resultados de sensibilidade, especificidade, VPP e VPN do auto-relato de diabetes mellitus gestacional. Dentre as 869 mulheres que portavam a carteira pré-natal com registro de glicemia, 37 apresentavam diabetes mellitus gestacional, correspondendo a uma prevalência de 4,3\% (IC95\%: 3,0-5,8). Declararam-se portadoras de diabetes mellitus gestacional 35 mulheres, das quais 27 eram casos verdadeiro-positivos da doença, correspondendo a uma sensibilidade de 72,9\% (IC95\%: 55,9-86,2). A especificidade do auto-relato de diabetes mellitus gestacional foi de 99\% (IC95\%: 98,1-99,6). Dentre as 35 que se declararam portadoras de diabetes mellitus gestacional, oito eram casos falso-positivos, correspondendo a um VPP do auto-relato de diabetes mellitus gestacional de 77,1\% (IC95\%: 59,9-89,6) (em cada quatro puérperas que se declararam diabéticas, uma não o era). O VPN foi de 98,8\% (IC95\%: 97,8-99,4) e a acurácia, 97,9\% (IC95\%: 96,7-98,7). A estatística kappa foi de $73,9 \%(\mathrm{p}<0,001)$, indicando que apenas $24,5 \%$ dos resultados concordantes deveram-se ao acaso.

Como não houve diferença estatisticamente significativa quanto à idade, cor da pele, IMC pré-gestacional e hospital do parto entre as mães que portavam e as que não portavam a carteira pré-natal, optou-se por expandir os resultados 
Distribuição das variáveis explanatórias entre as mães que levaram e as que não levaram a carteira de registro da gestante para o hospital. Pelotas, Rio Grande do Sul, Brasil.

\begin{tabular}{|c|c|c|c|c|c|c|}
\hline \multirow[t]{2}{*}{ Variáveis } & \multirow[t]{2}{*}{$\mathrm{N}$ total * } & \multicolumn{2}{|c|}{ Trouxeram a carteira } & \multicolumn{2}{|c|}{ Não trouxeram a carteira } & \multirow[t]{2}{*}{ Valor de $p$} \\
\hline & & $\mathbf{n}$ & $\%$ & $\mathbf{n}$ & $\%$ & \\
\hline \multicolumn{7}{|l|}{ Idade (anos) } \\
\hline$\leq 19$ & 180 & 158 & 18,3 & 22 & 13,1 & 0,15 \\
\hline $20-29$ & 533 & 447 & 51,9 & 86 & 51,2 & \\
\hline $30-49$ & 317 & 257 & 29,8 & 60 & 35,7 & \\
\hline \multicolumn{7}{|l|}{ Cor da pele da mãe } \\
\hline Branca & 714 & 592 & 67,7 & 122 & 70,9 & 0,29 \\
\hline Preta & 215 & 187 & 21,3 & 28 & 16,3 & \\
\hline Outra & 118 & 96 & 11,0 & 22 & 12,8 & \\
\hline \multicolumn{7}{|l|}{ IMC pré-gestacional (kg/m²) } \\
\hline$\leq 19$ & 160 & 122 & 18,5 & 38 & 27,7 & 0,07 \\
\hline $20-24$ & 412 & 344 & 52,2 & 68 & 49,6 & \\
\hline $25-29$ & 149 & 130 & 19,7 & 19 & 13,9 & \\
\hline$\geq 30$ & 75 & 63 & 9,6 & 12 & 8,8 & \\
\hline \multicolumn{7}{|l|}{ Hospital de nascimento } \\
\hline Santa Casa & 478 & 405 & 46,3 & 73 & 42,5 & 0,62 \\
\hline São Francisco de Paula & 549 & 454 & 51,9 & 95 & 55,2 & \\
\hline Miguel Pilcher & 20 & 16 & 1,8 & 4 & 2,3 & \\
\hline
\end{tabular}

* Diferenças nos $\mathrm{N}$ totais devem-se a perdas de informação para algumas variáveis.

\section{Tabela 2}

Propriedades do auto-relato de diabetes mellitus gestacional em comparação ao diagnóstico registrado de diabete mellitus gestacional na carteira pré-natal. Pelotas, Rio Grande do Sul, Brasil.

\begin{tabular}{lccc}
\hline & & Registro da carteira pré-natal \\
Auto-relato & Presente & Ausente & Total \\
\hline Presente & 27 (a) & 8 (b) & 35 \\
Ausente & $10(\mathrm{c})$ & $824(\mathrm{~d})$ & 834 \\
Total & 37 & 832 & 869 \\
\hline
\end{tabular}

Sensibilidade $=a /(a+c)=72,9 \%(I C 95 \%: 55,9-86,2)$; especificidade $=d /(b+d)=99,0 \%$ (IC95\%: 98,1-99,6); valor preditivo positivo $=a /(a+b)=77,1 \%(I C 95 \%: 59,9-89,6)$; valor preditivo negativo $=d /(c+d)=98,8 \%(I C 95 \%: 97,8-99,4) ;$ acurácia $=a+d /(a+b+c+d)=97,9 \%$ (IC95\%: 96,7-98,7); prevalência auto-referida $=(a+b) /(a+b+c+d)=4,0 \%(I C 95 \% 2,8-5,5)$; prevalência conforme padrão-ouro $=(a+c) /(a+b+c+d)=4,3 \%(I C 95 \%: 3,0-5,8)$; kappa $=0,74$.

encontrados para as 178 mães que não dispunham da carteira pré-natal no hospital. Para este grupo, calculou-se o número de mães que teriam diabetes mellitus gestacional auto-referida e o número conforme o padrão-ouro, resultando em um $\mathrm{N}$ total de 1.047. Com essa estratégia, houve um leve estreitamento dos intervalos de confiança para os parâmetros de sensibilidade e VPP (Tabela 3).
Entre as com IMC $\geq 30$, a prevalência de diabetes mellitus gestacional conforme registro na carteira pré-natal foi de 11,1\% (IC95\%: 0,4-21,9) Para investigar o efeito da idade e do IMC sobre a acurácia do auto-relato de diabetes mellitus gestacional, realizou-se a análise por regressão logística, tendo a variável "concordância" como desfecho. Devido ao fato de a diferença encontrada entre as médias dos pesos registrados e dos auto-referidos ser estatisticamente significativa, optou-se por usar o peso registrado para o cálculo do IMC, apesar da diminuição do tamanho da amostra. A medida de altura utilizada foi a autoreferida. Portanto, o IMC foi calculado por meio da divisão do peso registrado (desde que medido até o terceiro mês de gestação) pela altura autoreferida ao quadrado. A média do IMC pré-gestacional foi de 25,2 (DP $=4,9)$.

$\mathrm{Na}$ análise ajustada por regressão logística, as gestantes mais jovens, assim como as obesas, informaram mais acuradamente a presença ou ausência de diabetes mellitus gestacional, embora essas associações não tenham sido estatisticamente significativas.

A Figura 1 mostra os VPP e VPN do auto-relato conforme a prevalência de diabetes mellitus gestacional na população estudada. Quanto maior a prevalência de diabetes mellitus gestacional, 
maior o VPP do auto-relato. Para prevalências de $2,5 \%$, por exemplo, o VPP foi de $65,2 \%$, ao passo que, para prevalências de $6 \%$, foi de $82,3 \%$.

\section{Discussão}

Alguns estudos realizados para avaliar a validade do diagnóstico auto-referido de diabetes mellitus tipo 2 encontraram que essa medida tem alta acurácia, sendo muitas vezes superior ao registrado para outras doenças crônicas, como hipertensão arterial e hipercolesterolemia 18,19,20. Estudo realizado com gestantes latinas residentes nos Estados Unidos mostrou que, para questões comportamentais durante a gravidez, a concordância era baixa, mas para eventos médicos como diabetes mellitus gestacional, hipertensão arterial ou anemia era alta 21 . Também foi encontrada alta concordância para diabetes mellitus gestacional auto-referido no estudo norte-americano das enfermeiras 22 .

Entretanto, a acurácia do auto-relato depende de que os respondentes tenham conhecimento da sua situação, capacidade de recordar diagnósticos médicos e desejo de informar. A gravidez aumenta a chance de contato das mulheres com os serviços de saúde e, portanto, de diagnósticos de médicos 21 . Além disso, a consciência da responsabilidade com o feto leva a uma maior atenção da gestante a esses diagnósticos. O estudo de coorte de nascimentos realizado em Pelotas em 2004 mostrou que aproximadamente 99\% das mulheres comparecem a pelo menos uma consulta pré-natal 16 . No atual estudo, para $99 \%$ das mães que portavam a carteira de gestante, havia o registro de rastreamento para diabetes mellitus gestacional. Logo, em Pelotas, a oportunidade de conhecer o diagnóstico de diabetes mellitus gestacional, se presente, é alta.

Este estudo encontrou boa sensibilidade e alta especificidade do auto-relato de diabetes mellitus gestacional. O número observado de mulheres com essa doença conforme o padrãoouro foi inferior ao estimado por ocasião do cálculo do tamanho amostral. Essa redução levou a uma menor precisão na estimativa da sensibilidade do auto-relato de diabetes mellitus gestacional, que variou de 55,9\% a $86,2 \%$.

As puérperas que responderam negativamente à pergunta sobre diabetes mellitus gestacional e cujo registro de exames era compatível com diabetes mellitus gestacional (falso-negativas) apresentavam resultados de glicemia limítrofes (glicemia de jejum de 110mg/dL-126mg/dL). Tal achado pode estar indicando uma falha no processo diagnóstico de diabetes mellitus gestacional. Valores de glicemia de jejum dessa ordem
Tabela 3

Propriedades do auto-relato de diabetes mellitus gestacional em comparação ao diagnóstico registrado de diabete mellitus gestacional na carteira pré-natal, com expansão dos resultados para toda a amostra.

\begin{tabular}{|c|c|c|c|}
\hline \multirow[b]{2}{*}{ Auto-relato } & \multicolumn{3}{|c|}{ Registro da carteira pré-natal } \\
\hline & Presente & Ausente & Total \\
\hline Presente & 33 & 10 & 43 \\
\hline Ausente & 12 & 992 & 1.004 \\
\hline Total & 45 & 1.002 & 1.047 \\
\hline
\end{tabular}

Sensibilidade $=73,3 \%$ (IC95\%: 58,1-85,4); especificidade = 99\% (IC95\%: 98,2-99,5); valor preditivo positivo $=76,7 \%$ (IC95\%: 61,4-88,2); valor preditivo negativo $=98,8 \%$ (IC95\%: 97,999,4); acurácia = 97,9\% (IC95\%: 96,8-98,7); prevalência auto-referida = 4,1\% (IC95\%: 3,0-5,5); prevalência conforme padrão-ouro = 4,3\% (IC95\%: 3,2-5,7); kappa =0,71.

Figura 1

Valores preditivos positivos e negativos de diabetes mellitus gestacional auto-referido, conforme a prevalência de diabetes mellitus gestacional na população.

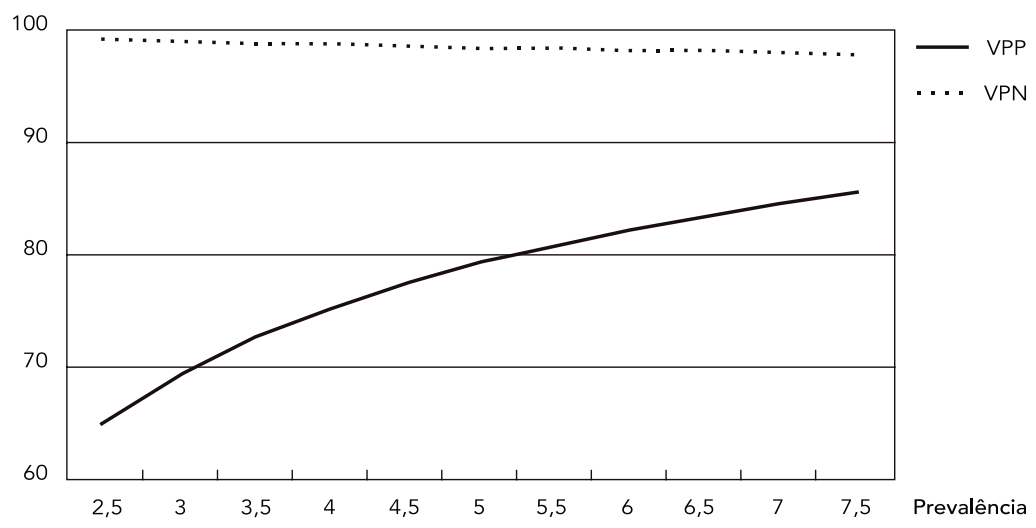

VPP: valor preditivo positivo; VPN: valor preditivo negativo

implicam a necessidade de repetição desse exame ou a realização de teste com sobrecarga de glicose. Por outro lado, as mulheres que disseram ter diabetes mellitus gestacional e não tinham na carteira exames com valores compatíveis com o diagnóstico (falso-positivos) apresentavam registros de glicemia de rastreamento elevada ( $\geq 90 \mathrm{mg} / \mathrm{dL}$ ), com posterior repetição do mesmo exame, sem a realização de teste de tolerância a glicose. Além dessa possível falha no procedimento diagnóstico, tal achado revela também indícios de uma possível mudança de comportamento durante a gestação. $\mathrm{O}$ achado de glice- 
mia elevada no rastreamento pode ter levado o profissional a recomendar mudanças do estilo de vida da gestante (mudanças alimentares e estímulo à atividade física), antes de solicitar o exame diagnóstico confirmatório.

Em virtude de várias campanhas publicitárias, é de domínio público o conhecimento de que a obesidade é fator de risco para diabetes mellitus. Este estudo encontrou que $100 \%$ das obesas souberam referir corretamente sua condição em relação ao diabetes mellitus gestacional, reforçando a idéia de uma maior atenção, por parte tanto dos médicos, quanto das gestantes, na presença de fatores de risco para diabetes mellitus na gravidez.

Uma limitação deste estudo pode ter sido o padrão-ouro utilizado. Embora o Ministério da Saúde norteie os procedimentos de rastreamento e diagnóstico do diabetes mellitus gestacional por meio de um protocolo, este nem sempre é seguido pelos profissionais de saúde 15. É possível que, se a adesão dos profissionais ao protocolo fosse melhor, as proporções de casos falso-positivos e falso-negativos seriam reduzidas, com conseqüente aumento da acurácia do diagnóstico auto-referido. É importante salientar, no entanto, que quase $100 \%$ das mães que levaram a carteira de gestante para o hospital tinham o registro de rastreamento para diabetes mellitus gestacional. É possível que as mães que levaram a carteira de pré-natal para o hospital tenham recebido um pré-natal de melhor qualidade do que as que não levaram. Por sua vez, as características examinadas no atual estudo (idade, cor da pele, IMC pré-gestacional e hospital do parto) que se associam com as condições sócio-econômicas da mulher não mostraram diferença estatisticamente significativa entre quem levou e quem não levou a carteira para o hospital.

Outra limitação diz respeito à capacidade de generalização dos resultados do estudo. O diagnóstico auto-referido de diabetes mellitus gestacional mostrou-se válido no pós-parto imedia- to, todavia não é possível afirmar que acurácia semelhante seria obtida caso as mães tivessem sido entrevistadas em um período mais distante da gestação. A capacidade de generalização dos resultados deste estudo deverá ser considerada com cuidado, pois dependerá da população de mulheres entre as quais o diagnóstico auto-referido de diabetes mellitus gestacional for investigado. Fatores como a qualidade da atenção pré-natal recebida, adesão dos profissionais ao rastreamento de diabetes mellitus gestacional, escolaridade materna, capacidade materna de recordação do diagnóstico médico recebido durante a gestação, bem como a valorização desse diagnóstico, deverão influenciar a validade do auto-relato em estudos conduzidos em contextos sócio-culturais distintos.

Em suma, os resultados desta investigação mostram que, para a população estudada, a utilização da informação de diabetes mellitus gestacional auto-referida é válida, com alta especificidade e boa sensibilidade. No entanto, há necessidade de maior conscientização dos profissionais de saúde com relação ao seguimento do protocolo de rastreamento e diagnóstico de diabetes mellitus gestacional.

A gravidez é uma situação de estresse fisiológico, podendo ser o momento mais precoce na vida da mulher para detectar aquelas em risco do desenvolvimento de diabetes mellitus no futuro. Essa detecção é passível de medidas preventivas, não somente em relação a uma gravidez subseqüente, mas também em relação à saúde da mulher ao longo da idade adulta e da velhice.

Portanto, rastreamento cuidadoso na gestação é uma importante oportunidade para identificar mulheres em maior risco de desenvolver diabetes mellitus no futuro. O rastreamento adequado, seguido de um auto-relato válido, como demonstrado no atual estudo, permitirão que estudos que se baseiam no diagnóstico auto-referido de diabetes mellitus gestacional possam ser conduzidos sem prejuízo de sua validade interna. 


\section{Resumo}

Pesquisas com base no auto-relato de fatores de risco ou morbidade são úteis em saúde pública, principalmente pelo baixo custo e facilidade de coleta, no entanto a acurácia dos dados coletados é importante para a validade interna da investigação. O objetivo deste estudo foi avaliar a concordância entre o auto-relato de diabete mellitus gestacional e registros do cartão da gestante (padrão-ouro). A concordância foi medida por meio da sensibilidade, especificidade, acurácia, estatística kappa e intervalos de 95\% de confiança (IC95\%). De julho a dezembro de 2007, todas as mulheres que tiveram filhos em três maternidades de Pelotas, Rio Grande do Sul, Brasil, foram visitadas no pós-parto imediato. Entrevistaram-se 1.047 mulheres, das quais 872 portavam a carteira de gestante; destas, 869 possuíam registro de glicemia. A média de idade foi 26,7 anos. A prevalência de diabetes mellitus gestacional conforme padrão-ouro foi 4,3\% (IC95\%: 3,0-5,8) e auto-referida, 4\% (IC95\%: 2,8-5,5). A sensibilidade do auto-relato de diabetes mellitus gestacional foi 72,9\% (IC95\%: 55,9-86,2); especificidade, 99\% (IC95\%: 98,199,6); acurácia, 97,9\% (IC95\%: 96,7-98,7); kappa, 74\%. Para esta população, auto-relato de diabetes mellitus gestacional é uma informação válida e pode ser usada em estudos de base populacional.

Diabetes Gestacional; Bem-Estar Materno; Prevalência

\section{Referências}

1. Centers for Disease Control and Prevention. Selfreported prevalence of diabetes among Hispanics - United States, 1994-1997. MMWR Morb Mortal Wkly Rep 1999; 48:8-12.

2. Centers for Disease Control and Prevention. Trends in the prevalence and incidence of self-reported diabetes mellitus - United States, 1980-1994. MMWR Morb Mortal Wkly Rep 1997; 46:1014-8.

3. Centers for Disease Control and Prevention. Trends in the prevalence and incidence of self-reported diabetes mellitus-United States, 1980-1994. JAMA 1997; 278:1564-5.

4. Borrell LN, Crawford ND, Dailo FJ. Race/ethnicity and self-reported diabetes among adults in the National Health Interview Survey: 2000-2003. Public Health Rep 2007; 122:616-25.

5. Bays HE, Bazata DD, Clark NG, Gavin 3rd JR, Green AJ, Lewis SJ, et al. Prevalence of self-reported diagnosis of diabetes mellitus and associated risk factors in a national survey in the US population: SHIELD (Study to Help Improve Early evaluation and management of risk factors Leading to Diabetes). BMC Public Health 2007; 7:277.

\section{Colaboradores}

M. A. S. O. Dode participou do projeto, revisão bibliográfica, elaboração do trabalho de campo, análise dos dados, discussão dos resultados e redação do manuscrito. I. S. Santos orientou a realização de todas as etapas da pesquisa e revisou o artigo.
6. Iunes M, Franco LJ, Wakisaka K, Iochida LC, Osiro $\mathrm{K}$, Hirai AT, et al. Self-reported prevalence of noninsulin-dependent diabetes mellitus in the 1st (Issei) and 2nd (Nisei) generation of Japanese-Brazilians over 40 years of age. Diabetes Res Clin Pract 1994;24 Suppl:S53-7.

7. Tang M, Chen Y, Krewski D. Gender-related differences in the association between socioeconomic status and self-reported diabetes. Int J Epidemiol 2003; 32:381-5.

8. Goldenberg P, Franco LJ, Pagliaro H, Silva RS, Santos CA. Diabetes mellitus auto-referido no Município de São Paulo: prevalência e desigualdade. Cad Saúde Pública 1996; 12:37-45.

9. Gikas A, Sotiropoulos A, Panagiotakos D, Peppas T, Skliros E, Pappas S. Prevalence, and associated risk factors, of self-reported diabetes mellitus in a sample of adult urban population in Greece: MEDICAL Exit Poll Research in Salamis (MEDICAL EXPRESS 2002). BMC Public Health 2004; 4:2.

10. Reti SR, Goodyear-Smith FA, McCormick RI. Selfreported diabetes in Northland, New Zealand. Diabetes Care 2005; 28:1258-9. 
11. Dallo FJ, Borrell LN. Self-reported diabetes and hypertension among Arab Americans in the United States. Ethn Dis 2006; 16:699-705.

12. Mohan V, Mathur P, Deepa R, Deepa M, Shukla DK, Menon GR, et al. Urban rural differences in prevalence of self-reported diabetes in India-The WHO-ICMR Indian NCD risk factor surveillance. Diabetes Res Clin Pract 2008; 80:159-68.

13. Welborn TA, Knuiman MW, Bartholomew HC, Whittall DE. 1989-90 National Health Survey: prevalence of self-reported diabetes in Australia. Med J Aust 1995; 163:129-32.

14. World Health Organization. Definition, diagnosis and classification of diabetes mellitus and its complications. Report of a WHO consultation. Geneva: World Health Organization; 1999. (WHO Technical Report Series)

15. Schirmer J. Assistência pré-natal: manual técnico. Brasília: Secretaria de Políticas de Saúde, Ministério da Saúde; 2000 .

16. Santos IS, Barros AJD, Matijasevich A, Tomasi E, Medeiros RS, Domingues MR, et al. Mothers and their pregnancies: a comparison of three population-based cohorts in Southern Brazil. Cad Saúde Pública 2008; 24 Suppl 3:S381-9.
17. Schmidt MI, Matos MC, Reichelt AJ, Forti AC, Lima L, Duncan BB. Prevalence of gestational diabetes mellitus - do the new WHO criteria make a difference? Brazilian Gestational Diabetes Study Group. Diabet Med 2000; 17:376-80.

18. El Fakiri F, Bruijnzeels MA, Hoes AW. No evidence for marked ethnic differences in accuracy of selfreported diabetes, hypertension, and hypercholesterolemia. J Clin Epidemiol 2007; 60:1271-9.

19. Ngo DL, Marshall LM, Howard RN, Woodward JA Southwick K, Hedberg K. Agreement between selfreported information and medical claims data on diagnosed diabetes in Oregon's Medicaid population. J Public Health Manag Pract 2003; 9:542-4.

20. Pendleton N, Clague JE, Cobain M, Thethi K, Jones M, Horan MA, et al. Relationship between self-reported prevalence of diabetes mellitus using the Cornell Medical Index (CMI) and prevalence determined by glycosylated hemoglobin (HbA(1c)) in an elderly community-dwelling population. Arch Gerontol Geriatr 2005; 41:289-96.

21. Hessol NA, Missett B, Fuentes-Afflick E. Lower agreement on behavioral factors than on medical conditions in self-reported data among pregnant Latina women. Arch Med Res 2004; 35:241-5.

22. Solomon CG, Willett WC, Rich-Edwards J, Hunter DJ, Stampfer MJ, Colditz GA, et al. Variability in diagnostic evaluation and criteria for gestational diabetes. Diabetes Care 1996; 19:12-6.

Recebido em 25/Abr/2008

Versão final reapresentada em $17 / \mathrm{Jul} / 2008$ Aprovado em 12/Ago/2008 